\title{
Room-temperature stabilization of antiferromagnetic skyrmions in synthetic antiferromagnets
}

\author{
William Legrand,, ${ }^{\text {}}$ Davide Maccariello, ${ }^{1}$ Fernando Ajejas, ${ }^{1}$ Sophie Collin, ${ }^{1}$ Aymeric \\ Vecchiola, ${ }^{1}$ Karim Bouzehouane, ${ }^{1}$ Nicolas Reyren, ${ }^{1}$ Vincent Cros, ${ }^{1}$ † and Albert Fert ${ }^{1}$ \\ ${ }^{1}$ Unité Mixte de Physique, CNRS, Thales, Univ. Paris-Sud, \\ Université Paris-Saclay, Palaiseau 91767, France
}

(Dated: July 17, 2019)

\begin{abstract}
Room-temperature skyrmions in ferromagnetic films and multilayers show promise for encoding information bits in new computing technologies. Despite recent progress, ferromagnetic order generates dipolar fields that prevent ultrasmall skyrmion sizes, and allows a transverse deflection of moving skyrmions that hinders their efficient manipulation. Antiferromagnetic skyrmions could lift these limitations. Here we demonstrate that room-temperature antiferromagnetic skyrmions can be stabilized in synthetic antiferromagnets (SAFs), in which perpendicular magnetic anisotropy (PMA), antiferromagnetic coupling and chiral order can be adjusted concurrently. Utilizing interlayer electronic coupling to an adjacent bias layer (BL), we demonstrate that spin-spiral states obtained in a SAF with vanishing PMA can be turned into isolated antiferromagnetic skyrmions. We also provide model-based estimates of skyrmion size and stability, showing that room-temperature antiferromagnetic skyrmions below $10 \mathrm{~nm}$ in radius can be anticipated in further optimized SAFs. Antiferromagnetic skyrmions in SAFs may thus solve major issues associated with ferromagnetic skyrmions for low-power spintronic devices.
\end{abstract}

* william.legrand@cnrs-thales.fr

$\dagger$ vincent.cros@cnrs-thales.fr 
Magnetic skyrmions are localized two-dimensional spin textures possessing a non-trivial topo$\log$ [1, 2]. They have triggered renewed interest in recent years since the experimental discovery of Dzyaloshinskii-Moriya interaction (DMI) [3, 4] at the interfaces of systems with broken inversion symmetry [5]. The interfacial DMI is a form of antisymmetric exchange interaction that establishes a preferred chirality for spin textures [6], making it a key ingredient for the formation of skyrmions in two-dimensional magnetic systems and nanoscale thin film multilayers [7], which has tremendously extended their potential range of applications. Isolated skyrmions can be obtained as metastable states in ferromagnets under external magnetic fields [8], can be nucleated, displaced and annihilated using current pulses [9-13], along with being detectable electrically [14, 15]. Provided that smaller skyrmion sizes (from the $100 \mathrm{~nm}$ to the $10 \mathrm{~nm}$ range) can be obtained and more reliable current-induced motion (faster velocities combined with further reduced depinning currents) can be achieved [16], all these possibilities are extremely promising for the conception of skyrmion-based nanodevices with dense integration and low energy cost.

The two most recurrent difficulties encountered so far with skyrmions hosted in ferromagnets are that dipolar interactions forbid ultrasmall skyrmion sizes [17] and that they make it extremely difficult to stabilize individual skyrmions without assistance from external magnetic fields [9, 18, 19]. In antiferromagnets, two coupled equivalent magnetic subsystems align antiparallel to each other with no net magnetic moment, thus generating vanishing dipolar fields. This property is foreseen as extremely interesting for skyrmions [20] as it could solve the two issues above. Furthermore, a consequence of the topology of ferromagnetic skyrmions on their dynamics is their deflection with respect to the direction of any driving force, known as the skyrmion Hall effect [21 23]. In contrast, the skyrmion Hall effect is compensated in antiferromagnets, which shall allow efficient skyrmion motion along the driving force direction [20, 24], crucial for applications such as skyrmion-based magnetic memory lines [16, 25]. With all these points in mind, understanding how to stabilize antiferromagnetic skyrmions constitutes the first step of a fundamental yet technologically-relevant investigation. A first illustration of their advantages has been recently obtained in ferrimagnetic materials, which are partially compensated ferromagnets [26, 27]. However, it might be difficult to control and detect noncollinear antiferromagnetic order in perfectly compensated antiferromagnets, as well as to control DMI in them, which is nonetheless essential in stabilizing skyrmions.

SAFs are substitutes for antiferromagnets that are composed of nm-thick ferromagnetic layers coupled antiferromagnetically through a non-magnetic spacer layer, by Ruderman-Kittel-KasuyaYoshida (RKKY) type interlayer electronic coupling [28]. Exactly as antiferromagnets, SAFs provide vanishing long-range dipolar interactions. However, because the two subsystems are separated 
by a spacer of around $1 \mathrm{~nm}$, small, but measurable, local dipolar fields can be present, which would facilitate the study of antiferromagnetic skyrmions hosted in SAFs. Besides, the DMI in SAFs can be conveniently controlled employing usual heavy-metal/ferromagnet interfaces, while theoretical studies of skyrmion motion in SAFs have revealed the possibility of driving them efficiently using spin-orbit torques [17, 29]. Exactly as antiferromagnetic skyrmions in antiferromagnets, antiferromagnetic skyrmions in SAFs would have many advantages over conventional, ferromagnetic skyrmions, and in addition may prove easier to study than in antiferromagnets. However, antiferromagnetic skyrmions in SAFs remain to be experimentally demonstrated.

Here, we explore how to stabilize antiferromagnetic skyrmions, and observe them experimentally in SAFs designed for this purpose. We show that in $[\mathrm{Pt} / \mathrm{Co} / \mathrm{Ru}]$-based multilayers it is possible to obtain concurrently all required controllable magnetic interactions. Adjusting their relative strengths by balancing individual layer thicknesses, we exhibit the stabilization of spin-spiral textures, which can be turned into zero-field individual antiferromagnetic skyrmions by coupling to an adjacent biasing multilayer. Our observation of antiferromagnetic skyrmions is consistent with model-based estimations of their size and stability. We believe that these results represent an important step towards the development of more advanced systems, exploiting even smaller skyrmions and without the requirement for external fields, bringing skyrmions closer to applications.

\section{ANTIFERROMAGNETIC SPIN-SPIRALS AND SKYRMIONS}

We first describe our approach in order to obtain antiferromagnetic skyrmions in SAFs. The antiferromagnetic coupling between two layers is expressed by the energy density per unit surface $E_{\mathrm{RKKY}}=J \mathbf{m}_{\mathbf{1}} \cdot \mathbf{m}_{\mathbf{2}}$, with $J$ the coupling energy (density), antiferromagnetic for $J>0$, and $\mathbf{m}_{\mathbf{1}}, \mathbf{m}_{\mathbf{2}}$ the magnetization directions in the bottom and top ferromagnetic layers of the SAF, respectively. Each magnetic layer of a perpendicular SAF is usually designed to possess a strong anisotropy and thus a uniform magnetization [30]. The ground state of such a SAF is the configuration in which $\mathbf{m}_{\mathbf{1}}$ and $\mathbf{m}_{\mathbf{2}}$ are antiparallel along the $z$ direction (perpendicular to the plane of the layers), represented in Figs. 17a and 1 b.

In case of negligible anisotropy, there is no longer an interaction that prevents the magnetic moments to rotate through the plane of the layers. Considering a SAF with significant DMI, it is even energetically favorable to obtain a non-collinear order where the magnetization in the layers rotates continuously, that is, a spin-spiral (Fig.117). A spin-spiral would form in the present nm-thick magnetic layers due to the almost complete compensation of the long-range dipolar 
interactions, which thus do not promote perpendicularly magnetized domains. Such spin-spiral state is otherwise only possible in atomically thin magnetic layers at low temperatures, for which the dipolar interactions are negligible [31]. Almost perfect spin-spirals without long-range order correlation, as illustrated in Fig. 11d, are expected when DMI, but no anisotropy, are present, which we aim to demonstrate experimentally.

Furthermore, we can examine how this configuration evolves under a biasing interaction. Due to compensation of the magnetization, SAFs remain nearly unaffected by moderate magnetic fields, and thus cannot be biased using external fields. However, we can obtain a biasing interaction by inserting a third magnetic layer, providing additional interlayer electronic coupling. Considering for example such layer (a BL) with uniform magnetization $\mathbf{m}_{\mathbf{b}}=+\mathbf{z}$ coupled ferromagnetically to the bottom layer of the SAF only, the parts of the spin-spiral with $\mathbf{m}_{\mathbf{1}}=+\mathbf{z}$ are promoted and expand at the expense of the parts with $\mathbf{m}_{\mathbf{1}}=-\mathbf{z}$ (Fig. 17). For a strong enough biasing interaction, antiferromagnetic skyrmions (defined as isolated, circular antiferromagnetic domains exhibiting a punctual center) can be formed, see Fig. 1F. In the following, we describe how we can build such a multilayered BL-SAF system to host antiferromagnetic skyrmions.

\section{TUNING PMA, DMI AND ANTIFERROMAGNETIC/BIAS COUPLINGS}

The key required magnetic properties are, namely, a strong antiferromagnetic coupling, a vanishing effective PMA, a significant DMI, and a biasing interaction. To combine all these features concurrently in a $\mathrm{SAF}$, we have selected a $[\mathrm{Pt} / \mathrm{Co} / \mathrm{Ru}]$-based multilayer deposited by sputtering (see Methods). The choice of a trilayer, breaking the inversion symmetry along the stacking direction, enables interfacial DMI, while Pt/Co interfaces provide the largest DMI found so far in sputtered systems [32], as well as significant PMA. The addition of a second interface with Ru does not degrade the DMI [33]. In $\mathrm{Co} / \mathrm{Ru} / \mathrm{Co}$ multilayers, the Ru layer provides an RKKY coupling between the Co layers, whose sign obeys to an oscillatory behavior depending on its thickness $t_{\mathrm{Ru}}$ [34, with an antiferromagnetic peak at around $0.8 \mathrm{~nm}$. Inserting thin Pt layers between Co and $\mathrm{Ru}$ layers still allows to transmit the RKKY coupling, yet reducing its strength, which allows to finely tune the RKKY coupling [35] and provides enhanced PMA [36, 37]. For all these reasons, $[\mathrm{Pt} / \mathrm{Co} / \mathrm{Ru}]$ multilayers constitute a very suitable system for our purpose.

The DMI magnitude $D$, RKKY coupling field $\mu_{0} H_{\mathrm{RKKY}}$ and effective PMA field $\mu_{0} H_{\text {eff }}$, all sharing an interfacial origin for the Co layers here, exhibit a $1 / t_{\text {Co }}$ dependence on Co layer thickness

$t_{\mathrm{Co}}$, with a negative offset at infinite thickness for PMA field only (see Methods). By increasing 
$t_{\text {Co }}$, we can obtain SAFs with no effective PMA, but significant DMI and RKKY coupling. As we demonstrate below, such SAFs can host spin-spirals as the ones in Figs. 11 and 1 d. By developing a tunable BL compatible with this SAF, we further obtain the possibility of forming antiferromagnetic skyrmions as in Figs. 1p and 1f. Hereafter, we present room-temperature characterization of different series of $[\mathrm{Pt} / \mathrm{Co} / \mathrm{Ru}]$ multilayers, aiming at optimizing BL-SAF properties for antiferromagnetic skyrmions. For each SAF, the values of $\mu_{0} H_{\text {eff }}$ and $\mu_{0} H_{\mathrm{RKKY}}$ can be determined following the measurement method described in Supplementary Note 1. We first investigate the dependence of the RKKY coupling on layer thicknesses $t_{\mathrm{Ru}}$ and $t_{\mathrm{Pt}}$, and find the optimal composition to obtain robust spin-spirals and eventually antiferromagnetic skyrmions. After this, we find the Co layer thickness required to cancel effective PMA, before we describe the BL and how to modulate its coupling strength.

We have deposited two series of multilayers, $\left[\operatorname{Pt}(0.8 \mathrm{~nm}) / \mathrm{Co}(0.8 \mathrm{~nm}) / \mathrm{Ru}\left(t_{\mathrm{Ru}}\right)\right]_{4}$, with $t_{\mathrm{Ru}}$ ranging $0.7-0.9 \mathrm{~nm}$ and $\left[\mathrm{Pt}\left(t_{\mathrm{Pt}}\right) / \mathrm{Co}(0.8 \mathrm{~nm}) / \mathrm{Ru}(0.8 \mathrm{~nm})\right]_{4}$, with $t_{\mathrm{Pt}}$ ranging $0.5-1.0 \mathrm{~nm}$, for which $\mu_{0} H_{\mathrm{RKKY}}$ is shown as a function of $t_{\mathrm{Ru}}$ and $t_{\mathrm{Pt}}$ in Figs. $2 \mathrm{a}$ and $2 \mathrm{~b}$, respectively. It is maximized for $t_{\mathrm{Ru}}$ around $0.7-0.75 \mathrm{~nm}$ (Fig. 2a): due to the insertion of Pt layers, the position of the antiferromagnetic coupling peak is shifted from its usual $\mathrm{Ru}$ thickness of $0.8 \mathrm{~nm}$ for $\mathrm{Co} / \mathrm{Ru} / \mathrm{Co}$, to increasingly small $\mathrm{Ru}$ thicknesses for increasingly large $t_{\mathrm{Pt}}$ [36]. The exponential decrease of the RKKY coupling with $t_{\mathrm{Pt}}$, due to the attenuation of the transmitted electrons [37], is confirmed (Fig. 2b). We find optimal conditions for PMA, DMI and RKKY coupling, with $J=0.115 \mathrm{~mJ} \mathrm{~m}^{-2}$, for the combination $[\mathrm{Pt}(0.6 \mathrm{~nm}) / \mathrm{Co} / \mathrm{Ru}(0.75 \mathrm{~nm})]$ (see Methods).

We now study both effective PMA and RKKY coupling modulated by $t_{\mathrm{Co}}$, keeping fixed $t_{\mathrm{Ru}}$ and $t_{\mathrm{Pt}}$ (see Supplementary Note 1 for measurements and associated behaviors). As shown in Figs. 2 2 and $2 \mathrm{~d}$, both terms follow the expected inverse dependence and $\mu_{0} H_{\mathrm{eff}}=0$ around $t_{\mathrm{Co}}=$ $1.49 \mathrm{~nm}$ (see Fig. 25), at which thickness the SAF undergoes spin reorientation transition from out-of-plane to in-plane. At this particular Co thickness, SAFs should therefore reproduce the conditions required for obtaining spin-spiral textures.

Eventually, we need to optimize the BL properties to exhibit a significant coercivity supported by PMA. As appears later, the ability to tune the bias coupling is also essential to stabilize antiferromagnetic skyrmions in SAFs, as it provides the analogous of the external field control in common ferromagnetic systems. We thus rely on a single, symmetrical Pt/Co multilayer [38] for the BL. The reasons for this choice and optimization of the BL are detailed in Supplementary Note 1. We finally selected $[\operatorname{Pt}(0.45 \mathrm{~nm}) / \mathrm{Co}(0.6 \mathrm{~nm})]_{4}$ for the BL under our SAF, whose out-of-plane magnetization loop, exhibiting full coercivity at zero field, is displayed in Fig. 2e. Our BL-SAF is 
finally $[\mathrm{Pt}(0.45 \mathrm{~nm}) / \mathrm{Co}(0.6 \mathrm{~nm})]_{4} / \mathrm{Pt}\left(t_{\mathrm{Pt}, \mathrm{BL}}\right) / \mathrm{SAF}$, inside which the strength of the coupling field $\mu_{0} H_{\text {bias }}$ between the $\mathrm{BL}$ and the $\mathrm{SAF}$ can be tuned by varying the intermediate Pt layer thickness $t_{\mathrm{Pt}, \mathrm{BL}}$ [39], as appears in Fig. 2: $\left(\mu_{0} H_{\text {bias }}\right.$ is measured using a different multilayer structure, see Supplementary Note 2).

\section{OBSERVING ANTIFERROMAGNETIC SPIN-SPIRALS AND SKYRMIONS}

Exploiting above optimization of the multilayer properties, we now turn to the observation of the resulting magnetic configurations in three different types of SAFs. We select (i) a SAF possessing a significant effective PMA (Fig. 3a), (ii) a SAF with a vanishing effective PMA (Fig. 3b), and (iii) a SAF combining a vanishing effective PMA and a BL (Fig. 3r), see Supplementary Note 1 for their magnetic characterization. These three systems constitute the experimental realization of the ones we have introduced in Fig. 1. Images are acquired at room temperature and without external field by Magnetic Force Microscopy (MFM), under vacuum to get enhanced sensitivity (see Methods).

In Fig. 34, we display such MFM image obtained for the SAF with PMA. Only a couple of domains separated by lines are present in a $4 \mu \mathrm{m} \times 3 \mu \mathrm{m}$ image. These domains are, as expected for this SAF, uniform antiferromagnetic configurations, similar to the representations of Figs. 1 1 a and 1b. They generate no fields, and therefore show no contrast. On the contrary, the narrow domain walls separating the antiferromagnetic domains generate non-canceling dipolar fields that appear as curved lines in the image, due to the small separation between the two compensating magnetic layers [40].

In Fig. 3e, we display the zero-field MFM image recorded for the SAF with vanishing effective PMA. Intertwined labyrinthine domains can be observed, which we assign to the presence of spinspirals looking similar to the magnetic configuration predicted by simulations in Figs. 1; and 1 $\mathrm{d}$. The periodicity of the spin-spirals observed here is about 260-300 nm. Neglecting any dipolar effects as they almost completely compensate here, the periodicity of spin-spirals is predicted to be $4 \pi A / D$, where $A$ is the Heisenberg exchange parameter (see Supplementary Note 3). Considering $A=10 \mathrm{pJ} \mathrm{m}^{-1}\left[18\right.$, we can estimate from this periodicity that $D=0.5 \mathrm{~mJ} \mathrm{~m}^{-2}$. This value is compatible with the one we can estimate from domain spacing measurements performed on similar, ferromagnetic multilayers, for which we find $D=0.76 \mathrm{~mJ} \mathrm{~m}^{-2}$ (Supplementary Note 4). The difference could originate in a weak anisotropy remaining in the SAF, as $1.47 \mathrm{~nm}$ may differ from the precise value of the spin reorientation thickness by a few percent. Note that the determination 
of the spin-spiral periodicity constitutes an independent measurement of the DMI amplitude for the present type of system.

Finally, we turn to the imaging of the optimized BL-SAF, which constituted the goal of our study. The BL is saturated under a positive field of $60 \mathrm{mT}$, before the MFM image in Fig. 3f is recorded at zero field, revealing isolated dots with clear dark contrast. We attribute them to the presence of antiferromagnetic skyrmions, based on a quantitative analysis of the expected MFM signal presented in Supplementary Notes 6-8. The dark contrast is here consistent with the expected positive sign of the dipolar fields resulting from antiferromagnetic skyrmions, due to the particular magnetization profiles adopted by them in each layer.

Additional images of the BL-SAF have been recorded under an external perpendicular field up to $100 \mathrm{mT}$ (Figs. 33-i). An evolution of the magnetic contrast and apparent size of the antiferromagnetic skyrmions with field is observed, followed by signal disappearance for fields around $100 \mathrm{mT}$, at which the annihilation of antiferromagnetic skyrmions occur because the external field overcomes the RKKY field. As detailed in Supplementary Note 6, the evolution recorded in this series of images under different fields is quantitatively consistent with the modeling of the stray field of antiferromagnetic skyrmions. We provide in Figs. 4a-d typical line sections of the spinspiral configuration and of antiferromagnetic skyrmions, corresponding to Figs. $3 \mathrm{e}-\mathrm{h}$, for which we determine contrast amplitude and apparent width (see Methods). These measures can be compared to predictions of MFM signal relying on micromagnetic simulations and stray field calculations (Supplementary Note 6). The results for contrast amplitude and apparent width are displayed in Figs. 4p and 4F, respectively. Comparing MFM data and calculations, the contrast levels match perfectly. A good agreement is also found for the evolution of the apparent skyrmion size (Fig. 4 d), despite some systematic underestimation. This difference is most probably due to an enlargement of the skyrmions because of their interaction with the strong moment of the magnetic tip during imaging, an effect that cannot be easily modeled. Antiferromagnetic skyrmions at zero field appear in Fig. 3 to measure around $50 \mathrm{~nm}$ in radius, however, this apparent width under MFM observation is different from their skyrmion radius $r_{\mathrm{sk}}$. The quantitative agreement between MFM contrast levels in experiments and simulations strongly supports that the radii of these antiferromagnetic skyrmions at zero field belong to the $20-30 \mathrm{~nm}$ range.

In ferromagnetic multilayers, skyrmions usually appear in a narrow region of applied external fields because of the detrimental role of the dipolar fields [9, 18, 19]. Here, because antiferromagnetic skyrmions are largely insensitive to dipolar fields, and only moderately affected by external fields (below the RKKY field limit), they remain stable under a large range of external fields including 
zero field $\left(\mu_{0} H_{\text {ext }}=0-60 \mathrm{mT}\right)$. Instead, antiferromagnetic skyrmion stability criterion is whether the suitable biasing field $\mu_{0} H_{\text {bias }}$ is obtained, verified here for $t_{\mathrm{Pt}, \mathrm{BL}}=2.2 \mathrm{~nm}$. For larger values of $\mu_{0} H_{\text {bias }}$ (smaller $t_{\mathrm{Pt}, \mathrm{BL}}$ ), we find that forming magnetic domains in the bottom layer of the SAF is disfavored and observe uniform configuration, while for lower values of $\mu_{0} H_{\text {bias }}$ (larger $t_{\mathrm{Pt}, \mathrm{BL}}$ ), we find that the spin-spiral state remains almost unaffected, due to insufficient coupling.

\section{PROPERTIES OF ANTIFERROMAGNETIC SKYRMIONS}

To better understand the influence of the different magnetic parameters on the antiferromagnetic skyrmion properties, we perform micromagnetic modeling of our BL-SAF antiferromagnetic skyrmions [41] and discuss how they are affected, in energy and size, by DMI and bias coupling strengths (see Methods). We first display the radial profiles of the vertical component of the magnetization $\left(m_{\mathrm{z}}\right)$ of the skyrmion in the bottom layer of the SAF, varying $\mu_{0} H_{\text {bias }}$ in the range 20-80 $\mathrm{mT}$ for $D=0.5 \mathrm{~mJ} \mathrm{~m}^{-2}$ (Fig. 5 a), and varying $D$ in the range $0.3-0.8 \mathrm{~mJ} \mathrm{~m}^{-2}$ for $\mu_{0} H_{\text {bias }}=$ $40 \mathrm{mT}$ (Fig. 5b). We analyze the profiles in both magnetic layers, which only slightly differ from each other, in Supplementary Note 9. The results of our modeling (lines in Figs. 5 a and 5b) show an excellent agreement with the results provided by the standard micromagnetic package $\mathrm{MuMax}^{3}$ [42] (circles in Figs. 5a and 5b). It appears that the size of the skyrmion reduces for increasing $\mu_{0} H_{\text {bias }}$ and increases for increasing $D$, because the bias coupling favors confinement within the surrounding uniform state, whereas the DMI favors the rotating magnetization of the skyrmion and its expansion.

A crucial issue regarding the scalability of room-temperature applications of skyrmions is their size-dependent thermal stability. We use here a technique allowing to approximate the skyrmion lifetimes from the energy barrier $E_{\mathrm{B}}$ preventing their isotropic collapse, within a micromagnetic model ( [17], see Methods). To obtain this annihilation barrier height, we first analyze how the different energy components evolve with the skyrmion radius $r_{\text {sk }}$ down to $3 \mathrm{~nm}$, as shown in Fig. 5c. As emphasized by the linear fitting of the different energy terms at low radius values, all terms decrease to zero for vanishing sizes, except exchange energy $E_{\mathrm{A}}$ [43]. By extrapolating $E_{\mathrm{A}}$ down to zero radius, we find around $E_{\mathrm{A}}(0)=24.5 A d$, with $d=2 t_{\mathrm{Co}}$ [17]. The energy barrier can then be extracted, within the approximation made by our simplified model (see Methods), from the radius dependence of the skyrmion energy $E_{\mathrm{sk}}$, which is the sum of all terms displayed in Fig. 5f. As shown in Fig. 5d, we compare $E_{\mathrm{A}}(0)$ to $E_{\mathrm{sk}}$ at the radius of minimal energy to get the energy barrier $E_{\mathrm{B}}$. Note that $E_{\mathrm{sk}}>0$ in all studied cases, which means that the uniform, 
antiferromagnetic state possesses a lower energy. Therefore, the antiferromagnetic skyrmions do not constitute the ground state of the system, but rather metastable states, as it is the case for isolated ferromagnetic skyrmions.

Finally, we study how $r_{\text {sk }}$ (Fig. 5e) and $E_{\mathrm{B}}$ (Fig. 5f) evolve together with $D$ and $\mu_{0} H_{\text {bias }}$. Antiferromagnetic skyrmions of similar sizes are found to be more stable for larger values of $D$. A thermal stability barrier higher than $40 k_{\mathrm{B}} T$ is typically required for long-term storage of skyrmions. An important result is that skyrmion sizes as small as around $10 \mathrm{~nm}$ can be reached with a satisfying thermal stability [17] in the present SAF bilayer, for sufficiently large values of $D$. For the antiferromagnetic skyrmions that we have observed, remembering that we estimate $D=0.5-$ $0.7 \mathrm{~mJ} \mathrm{~m}^{-2}$ and $\mu_{0} H_{\text {bias }}=50 \mathrm{mT}$, we deduce that the actual skyrmion radius should reach $25 \mathrm{~nm}$, in full agreement with the value deduced from the analysis and modeling of the MFM image of Fig. 3f and Supplementary Note 6.

\section{OUTLOOK}

The key advantages of the present BL-SAF based on $[\mathrm{Pt} / \mathrm{Co} / \mathrm{Ru}]$ multilayers, compared to the ferromagnetic multilayers utilized so far, are that external fields are no longer necessary to stabilize skyrmions, and even more, that the dipolar fields are largely compensated inside them. Contrary to ferromagnetic multilayers in which the accumulation of repeated magnetic layers has detrimental repercussions on the internal structure and stability of the skyrmions [44, it is thus in principle possible to accumulate several antiferromagnetically coupled pairs of layers without significantly affecting the structure of the skyrmions. In that respect, it seems even more judicious to increase the thermal stability of antiferromagnetic skyrmions by simply increasing the total number of magnetic layers [18]. Given that our $[\mathrm{Pt} / \mathrm{Co} / \mathrm{Ru}]$ system allows stacking identical layers with equivalent properties, we believe that obtaining similar, but more stable, antiferromagnetic skyrmions in multilayers made of more than 2 antiferromagnetically coupled layers does not present any obstacle, allowing to further reduce skyrmion sizes below $10 \mathrm{~nm}$ while keeping room-temperature stability. We show in Supplementary Note 11 the expected size and stability of skyrmions in SAFs with 4 antiferromagnetically coupled layers, which supports this statement.

We have shown that spin-spiral and antiferromagnetic skyrmion states can be detected by sensitive MFM imaging under low pressures. However, other means should be effective and could be studied to allow more applicable detection of the antiferromagnetic skyrmions, for example, through transverse conductivity electrical detection [14, 15] or using the non-collinear magnetoresistance 
[45, 46].

In Supplementary Note 12, we demonstrate the stability of these antiferromagnetic skyrmions up to $T=420 \mathrm{~K}$. This allows for a significant heating of the system, which provides the future possibility of injecting large current-pulses in order to induce skyrmion motion. Due to the compensation of the topological charge of the two skyrmions with identical saturation magnetization hosted in the bottom and top layers [17, 29], a straight and efficient motion is expected at any $T$. The stabilization of antiferromagnetic skyrmions in SAFs at room temperature and beyond opens many possibilities for future studies on magnetic skyrmions, and should rapidly contribute to answer the need for extremely small and mobile skyrmions required in many device applications. 


\section{REFERENCES}

[1] Bogdanov, A. N. \& Rößler, U. K. Chiral Symmetry Breaking in Magnetic Thin Films and Multilayers. Phys. Rev. Lett. 87, 037203 (2001).

[2] Rößler, U. K., Bogdanov, A. N. \& Pfleiderer, C. Spontaneous skyrmion ground states in magnetic metals. Nature 442, 797-801 (2006).

[3] Dzyaloshinsky, I. A thermodynamic theory of "weak" ferromagnetism of antiferromagnetics. J. Phys. Chem. Solids 4, 241-255 (1958).

[4] Moriya, T. Anisotropic Superexchange Interaction and Weak Ferromagnetism. Phys. Rev. 120, 91-98 (1960).

[5] Bode, M. et al. Chiral magnetic order at surfaces driven by inversion asymmetry. Nature 447, 190-193 (2007).

[6] Heide, M., Bihlmayer, G. \& Blügel, S. Dzyaloshinskii-Moriya interaction accounting for the orientation of magnetic domains in ultrathin films: Fe/W(110). Phys. Rev. B 78, 140403 (2008).

[7] Kiselev, N. S., Bogdanov, A. N., Schäfer, R. \& Rößler, U. K. Chiral skyrmions in thin magnetic films: new objects for magnetic storage technologies? J. Phys. D: Appl. Phys. 44, 392001 (2011).

[8] Romming, N. et al. Writing and deleting single magnetic skyrmions. Science 341, 636-639 (2013).

[9] Woo, S. et al. Observation of room-temperature magnetic skyrmions and their current-driven dynamics in ultrathin metallic ferromagnets. Nat. Mater. 15, 501-506 (2016).

[10] Legrand, W. et al. Room-Temperature Current-Induced Generation and Motion of sub-100 nm Skyrmions. Nano Lett. 17, 2703-2712 (2017).

[11] Büttner, F. et al. Field-free deterministic ultrafast creation of magnetic skyrmions by spin-orbit torques. Nat. Nanotech. 12, 1040-1044 (2017).

[12] Woo, S. et al. Deterministic creation and deletion of a single magnetic skyrmion observed by direct time-resolved X-ray microscopy. Nat. Electron. 1, 288-296 (2018).

[13] Hrabec, A. et al. Current-induced skyrmion generation and dynamics in symmetric bilayers. Nat. Commun. 8, 15765 (2017).

[14] Maccariello, D. et al. Electrical detection of single magnetic skyrmions in metallic multilayers at room temperature. Nat. Nanotech. 13, 233-237 (2018).

[15] Zeissler, K. et al. Discrete hall resistivity contribution from néel skyrmions in multilayer nanodiscs. Nature Nanotechnology 13, 1161-1166 (2018).

[16] Fert, A., Reyren, N. \& Cros, V. Magnetic skyrmions: advances in physics and potential applications. Nat. Rev. Mat. 2, 17031 (2017).

[17] Büttner, F., Lemesh, I. \& Beach, G. S. D. Theory of isolated magnetic skyrmions: From fundamentals to room temperature applications. Sci. Rep. 8, 4464 (2018).

[18] Moreau-Luchaire, C. et al. Additive interfacial chiral interaction in multilayers for stabilization of small individual skyrmions at room temperature. Nat. Nanotech. 11, 444-448 (2016). 
[19] Boulle, O. et al. Room-temperature chiral magnetic skyrmions in ultrathin magnetic nanostructures. Nat. Nanotech. 11, 449-454 (2016).

[20] Barker, J. \& Tretiakov, O. A. Static and Dynamical Properties of Antiferromagnetic Skyrmions in the Presence of Applied Current and Temperature. Phys. Rev. Lett. 116, 147203 (2016).

[21] Nagaosa, N. \& Tokura, Y. Topological properties and dynamics of magnetic skyrmions. Nat. Nanotech. 8, 899-911 (2013).

[22] Jiang, W. et al. Direct observation of the skyrmion Hall effect. Nat. Phys. 13, 162-169 (2017).

[23] Litzius, K. et al. Skyrmion Hall effect revealed by direct time-resolved X-ray microscopy. Nat. Phys. 13, 170-175 (2017).

[24] Zhang, X., Zhou, Y. \& Ezawa, M. Magnetic bilayer-skyrmions without skyrmion Hall effect. Nat. Commun. 7, 10293 (2016).

[25] Sampaio, J., Cros, V., Rohart, S., Thiaville, A. \& Fert, A. Nucleation, stability and current-induced motion of isolated magnetic skyrmions in nanostructures. Nat. Nanotech. 8, 839-844 (2013).

[26] Woo, S. et al. Current-driven dynamics and inhibition of the skyrmion Hall effect of ferrimagnetic skyrmions in GdFeCo films. Nat. Commun. 9, 959 (2018).

[27] Caretta, L. et al. Fast current-driven domain walls and small skyrmions in a compensated ferrimagnet. Nat. Nanotech. 13, 1154-1160 (2018).

[28] Parkin, S. S. P., Bhadra, R. \& Roche, K. P. Oscillatory magnetic exchange coupling through thin copper layers. Phys. Rev. Lett. 66, 2152-2155 (1991).

[29] Zhang, X., Zhou, Y. \& Ezawa, M. Antiferromagnetic Skyrmion: Stability, Creation and Manipulation. Sci. Rep. 6, 24795 (2016).

[30] Hellwig, O., Kirk, T. L., Kortright, J. B., Berger, A. \& Fullerton, E. E. A new phase diagram for layered antiferromagnetic films. Nat. Mater. 2, 112-116 (2003).

[31] Hervé, M. et al. Stabilizing spin spirals and isolated skyrmions at low magnetic field exploiting vanishing magnetic anisotropy. Nat. Commun. 9, 1015 (2018).

[32] Hrabec, A. et al. Measuring and tailoring the Dzyaloshinskii-Moriya interaction in perpendicularly magnetized thin films. Phys. Rev. B 90, 020402 (2014).

[33] Belmeguenai, M. et al. Interface Dzyaloshinskii-Moriya interaction in the interlayer antiferromagneticexchange coupled Pt/CoFeB/Ru/CoFeB systems. Phys. Rev. B 96, 144402 (2017).

[34] Bloemen, P. J. H., van Kesteren, H. W., Swagten, H. J. M. \& de Jonge, W. J. M. Oscillatory interlayer exchange coupling in Co/Ru multilayers and bilayers. Phys. Rev. B 50, 13505-13514 (1994).

[35] Lavrijsen, R. et al. Magnetic ratchet for three-dimensional spintronic memory and logic. Nature 493, 647-650 (2013).

[36] Bandiera, S., Sousa, R. C., Auffret, S., Rodmacq, B. \& Dieny, B. Enhancement of perpendicular magnetic anisotropy thanks to Pt insertions in synthetic antiferromagnets. Appl. Phys. Lett. 101, $072410(2012)$.

[37] Lavrijsen, R. et al. Tuning the interlayer exchange coupling between single perpendicularly magnetized 
CoFeB layers. Appl. Phys. Lett. 100, 052411 (2012).

[38] Yakushiji, K. et al. Ultrathin $\mathrm{Co} / \mathrm{Pt}$ and $\mathrm{Co} / \mathrm{Pd}$ superlattice films for MgO-based perpendicular magnetic tunnel junctions. Appl. Phys. Lett. 97, 232508 (2010).

[39] Moritz, J., Garcia, F., Toussaint, J. C., Dieny, B. \& Nozières, J. P. Orange peel coupling in multilayers with perpendicular magnetic anisotropy: Application to (Co/Pt)-based exchange-biased spin-valves. Europhys. Lett. 65, 123-129 (2004).

[40] Hellwig, O., Berger, A. \& Fullerton, E. E. Domain Walls in Antiferromagnetically Coupled Multilayer Films. Phys. Rev. Lett. 91, 197203 (2003).

[41] Legrand, W. et al. Modeling the Shape of Axisymmetric Skyrmions in Magnetic Multilayers. Phys. Rev. Appl. 10, 064042 (2018).

[42] Vansteenkiste, A. et al. The design and verification of MuMax3. AIP Adv. 4, 107133 (2014).

[43] Rohart, S., Miltat, J. \& Thiaville, A. Path to collapse for an isolated néel skyrmion. Phys. Rev. B 93, $214412(2016)$.

[44] Legrand, W. et al. Hybrid chiral domain walls and skyrmions in magnetic multilayers. Sci. Adv. 4, eaat0415 (2018).

[45] Hanneken, C. et al. Electrical detection of magnetic skyrmions by tunnelling non-collinear magnetoresistance. Nat. Nanotech. 10, 1039-1042 (2015).

[46] Crum, D. M. et al. Perpendicular reading of single confined magnetic skyrmions. Nat. Commun. 6, 8541 (2015).

[47] Bessarab, P. F. et al. Lifetime of racetrack skyrmions. Sci. Rep. 8, 3433 (2018).

[48] Bessarab, P. F. et al. Stability and lifetime of antiferromagnetic skyrmions. Phys. Rev. B 99, 140411 (2019).

[49] Wild, J. et al. Entropy-limited topological protection of skyrmions. Sci. Adv. 3, e1701704 (2017).

[50] Belavin, A. \& Polyakov, A. Metastable states of two-dimensional isotropic ferromagnets. JETP Lett. 22, 245-248 (1975). 


\section{METHODS}

Multilayers deposition and characterization. Multilayered SAFs have been fabricated by dc magnetron sputtering at room temperature on thermally oxidized silicon substrates, under Ar gas flow at a pressure of $0.25 \mathrm{~Pa}$, with calibrated deposition rates. Our choice is motivated by the fact that sputtering is an industry-compatible process with excellent reproducibility and control over the deposition rates, so that we are able to control the individual layer thicknesses with a precision better than $0.3 \AA$. Base pressure of the sputtering equipment was $5 \times 10^{-6} \mathrm{~Pa}$. All multilayers described in this study have been deposited on top of $\mathrm{Ta}(5 \mathrm{~nm}) / \mathrm{Pt}(8 \mathrm{~nm})$ buffers (denoted as Buff. in insets of Figs. 2 and 3), and capped with $\operatorname{Pt}(3 \mathrm{~nm})$ layers to prevent oxidation. The in-plane and out-of-plane magnetization measurements have been performed using an alternating gradient magnetometry setup.

In the Co layers, the intralayer dipolar fields generated by the local ferromagnetic order (shape anisotropy effect) actually reduce the PMA energy term $K_{\mathrm{u}}$ to an effective value $K_{\text {eff }}=K_{\mathrm{u}}-$ $\mu_{0} M_{\mathrm{s}}^{2} / 2$ (anisotropy field $\mu_{0} H_{\text {eff }}=2 K_{\text {eff }} / M_{\mathrm{s}}$ ), with $M_{\mathrm{s}}$ the saturation magnetization of Co. It is this value $K_{\text {eff }}$ that discriminates between the possible preferred magnetic orientations, perpendicular (towards $m_{\mathrm{z}}= \pm 1$, for $K_{\mathrm{eff}}>0$ ) or in-plane (towards $m_{\mathrm{z}}=0$, for $K_{\text {eff }}<0$ ). The offset $-\mu_{0} M_{\mathrm{S}}^{2} / 2$ allows to reach a vanishing effective PMA $\left(K_{\mathrm{eff}}=0\right)$ by increasing $t_{\mathrm{Co}}$, while keeping a significant DMI magnitude. Likewise, the RKKY coupling field $\mu_{0} H_{\mathrm{RKKY}}=2 J /\left(t_{\mathrm{Co}} M_{\mathrm{S}}\right)$, also linked to interfacial coupling $J$ by a $1 / t_{\text {Co }}$ dependence, hence remains significant for a vanishing effective PMA. We have to keep in mind that we aim at optimizing the RKKY coupling while still preserving strong DMI and PMA $K_{\mathrm{u}}$ (if $K_{\mathrm{u}}$ is too low, $K_{\text {eff }}$ would be negative and it would be impossible to reach $K_{\text {eff }}=0$ by tuning $t_{\mathrm{Co}}$ ). These two decrease sharply for $t_{\mathrm{Pt}}<0.6 \mathrm{~nm}$. Using this minimal Pt layer thickness $t_{\mathrm{Pt}}=0.6 \mathrm{~nm}$, the antiferromagnetic peak is located at $t_{\mathrm{Ru}}=$ $0.75 \mathrm{~nm}$.

MFM observation. The MFM observation of the SAFs has been performed in a setup under low pressure, of the order of $P=5 \mathrm{~Pa}$. Images were obtained using magnetic tips in a double pass tapping mode - lift mode, detecting the phase shift of the second pass after a topographic measurement and thus probing the magnetic field gradient along the vertical direction. Tips were fabricated in our laboratory by depositing a magnetic coating on commercial silicon tips with magnetic sputtering, whose thicknesses ranged 3-23 nm of CoFeB (see Supplementary Note 6), which we selected for their particularly low degree of perturbation on the magnetic configurations under observation, and improved signal-to-noise ratio, with quality factor $Q=1500$ and spring 
constant $k=0.4 \mathrm{~N} \mathrm{~m}^{-1}$.

Note that for either the unbiased or the biased SAFs with vanishing effective PMA, there is no contrast within the sensitivity of our microscope in any zero-field image performed without putting the microscope under vacuum to increase its sensitivity. On the contrary, even in single ferromagnetic layers, a clear contrast is routinely obtained with our MFM setup under ambient conditions for ferromagnetic stripe domains or skyrmion configurations. This supports that the much weaker magnetic signal observed in the present experiment does not originate from ferromagnetic but from compensated textures. Supplementary Note 5 reports imaging of various ferromagnetic textures or saturated configurations in the different elements constituting the BL-SAF, showing that the BL remains uniformly saturated at zero field and that we image the configuration in the SAF only; that we can exclude ferromagnetic skyrmions that would be located in a single layer; and that the small variations of contrast visible around the dark dots in Fig. 3f originate from a small background signal present in any system.

To be able to apply external perpendicular fields in our MFM setup under vacuum, the magnetic multilayers have been placed on top of tiny permanent magnets with different spacers, producing different calibrated values of fields, for which reason the same skyrmions could not be imaged at different magnetic fields. The multiple linecuts serve here as an uncertainty estimate for the population of skyrmions. Gaussian fits to the antiferromagnetic skyrmions data allow to extract a contrast level (amplitude of the Gaussian) and an apparent width (full-width at half maximum of the Gaussian), the later being comparable to the diameter $2 r_{\mathrm{sk}}$ of the skyrmions if the effects of the MFM imaging were neglected.

Micromagnetic simulations. To perform the modeling, we use a custom micromagnetic solver to find axisymmetric profiles of skyrmions by energy minimization [41], to which we add the RKKY coupling between the two layers. Parameters are $A=10 \mathrm{pJ} \mathrm{m}^{-1}$ for Heisenberg exchange, $D=0.2-0.8 \mathrm{~mJ} \mathrm{~m}^{-2}$ for DMI,$M_{\mathrm{s}}=1.2 \mathrm{MA} \mathrm{m}^{-1}$ for saturation magnetization of Co, $K_{\mathrm{u}}=$ $0.905 \mathrm{MJ} \mathrm{m}^{-3}$ (which corresponds to $K_{\text {eff }}=0 \mathrm{MJ} \mathrm{m}^{-3}$ ) for PMA energy, $\mu_{0} H_{\text {ext }}=0 \mathrm{mT}$ for the externally applied field, $J=0.115 \mathrm{~mJ} \mathrm{~m}^{-2}$ for RKKY coupling energy, Co thickness $t_{\mathrm{Co}}=1.47 \mathrm{~nm}$ and layers spacing $p=2.82 \mathrm{~nm}$ (which corresponds to the measured magnetic properties of our optimized $[\mathrm{Pt}(0.6) / \mathrm{Co}(1.47) / \mathrm{Ru}(0.75)]_{2} \mathrm{SAF}$ ), except stated otherwise. Initial simulations (see Fig. 1) have been performed using the micromagnetic code $\operatorname{MuMax}^{3}$ [42]. The cubic cell size was fixed at $1.47 \mathrm{~nm}$ in a grid of $1024 \times 1024 \times 4$ cells in which we have introduced the antiferromagnetic coupling. We used for Figs. 1 $1 \mathrm{a}$ and $1 \mathrm{~b} K_{\mathrm{u}}=1.025 \mathrm{MJ} \mathrm{m}^{-3}$, for Figs. 1 1 and 1 f $\mu_{0} H_{\text {bias }}=20 \mathrm{mT}$. The modeling of antiferromagnetic skyrmions, as shown in Fig. 5 , has been done with our own energy 
minimizer [41] after we checked that it was providing identical results with the micromagnetic code $\mathrm{MuMax}^{3}$. When no energy barrier is present, the skyrmion collapses and only a small energy related to boundary condition $\mathbf{m}(0)=-\mathbf{z}$ remains, corresponding to the gray color in diagrams of Figs. 5e and 5. To vary the size of the skyrmion for a single set of magnetic parameters (Fig. 5d), only one discretization element is imposed to have in-plane magnetization at given skyrmion radius $r_{\mathrm{sk}}$, hence fixing $r_{\mathrm{sk}}$ for this minimization. We have also checked the stability against anisotropic deformation of the skyrmions, by initializing the simulation with a stripe domain and verifying that it reduces into a skyrmion for all sets of parameters in Figs. 5e and 5f.

Assumptions for the thermal stability estimation. In order to thoroughly investigate the stability of antiferromagnetic skyrmions, we would need to find the minimal energy reaction path towards collapse into the uniform state by performing Nudged Elastic Band calculations [43, 47, 48, and to obtain the annihilation attempt frequency and energy barrier thereof. However this technique goes beyond the scope of our study as it requires the modeling of skyrmions down to few-atoms radii and thus requires an atomistic Hamiltonian description, which has been applied, so far, only to single atomic layers of two-dimensional systems, neglecting any thickness. A simpler approach to address this issue, at least partially, is to assume attempt frequencies found in the literature, and only consider the energy barrier $E_{\mathrm{B}}$ term. The lifetime $\tau$ of the skyrmions is linked to the energy barrier height $E_{\mathrm{B}}$ and temperature $T$ by the Arrhenius law, $1 / \tau=k(T) e^{-E_{\mathrm{B}} /\left(k_{\mathrm{B}} T\right)}$. In this relation, the stability timescale is set by the temperature-dependent prefactor $k(T)$ (attempt frequency), which can only be calculated from a precise knowledge of the minimum energy path, but to which we cannot access here. It has been shown that for the isotropic annihilation of isolated magnetic skyrmions in extended thin film, which we consider here, the attempt frequency is about $10^{9}-10^{10} \mathrm{~s}^{-1}$ [3]. It is also known that it may change with temperature, and drastically change by orders of magnitudes for other annihilation processes, or for skyrmions stabilized in other compounds such as chiral magnets [49]. Nevertheless assuming this value for the attempt frequency, we focus on the barrier height. Owing to the exponential dependence of the lifetime with barrier height, any misestimation of the attempt frequency would simply shift the required barriers by several $k_{\mathrm{B}} T$.

We define the size of the skyrmion $r_{\mathrm{sk}}$ as the radius at which $m_{\mathrm{z}}=0$ in the bottom layer. We consider that the continuous description of the magnetization inside the skyrmion, within the framework of the micromagnetic model, remains valid as long as $r_{\mathrm{sk}}>3 \mathrm{~nm}$. We do not perform calculations on smaller skyrmions, as it would mean that the magnetization orientation would change by more than $8^{\circ}$ between two atoms. Considering skyrmions of size $r_{\mathrm{sk}}>3 \mathrm{~nm}$, they are 
expected to keep axisymmetric profiles along the minimal energy path [43]. Note that actually the dipolar fields play some minor role in the overall skyrmion energy, despite the almost complete compensation of the magnetization (see Supplementary Note 10).

The value of $E_{\mathrm{A}}$ at $r_{\mathrm{sk}}=0$ that we interpolate is very close to $8 \pi A d$, the minimal exchange energy for a skyrmion having an integer topological charge in a continuous description [50]. This value is in between $27 A d$ that can be found by using a general skyrmion profile [17], and $22 \mathrm{Ad}$ found in Nudged Elastic Band calculations [43. This is because the radial magnetization profile is entirely free in our case, which relaxes partly the exchange energy below $27 A d$, but remains an axially symmetrical and continuous profile without any deformations, resulting in a value above $22 \mathrm{Ad}$. It is to be pointed out that the precise value of the exchange energy at annihilation should also depend on the interlayer exchange coupling, exact spin configuration, atomic structure of the layers and/or on potential inhomogeneity in the materials, and is therefore in any case an approximation by a couple $A d$, that is, a few $k_{\mathrm{B}} T$ at room temperature.

\section{DATA AVAILABILITY}

All relevant data presented in the manuscript and in the Supplementary information supporting the findings of this study are available from the corresponding authors upon request.

\section{ACKNOWLEDGMENTS}

The authors thank W. Akhtar, A. Finco, S. Chouaieb and V. Jacques for discussions about the magnetic imaging of SAFs. Financial support from the Agence Nationale de la Recherche, France, under grant agreement No. ANR-17-CE24-0025 (TOPSKY), the Horizon2020 Framework Programme of the European Commission, under FET-Proactive Grant agreement No. 824123 (SKYTOP) and FET-Open grant agreement No. 665095 (MAGicSky), and the DARPA TEE program, through grant MIPR \# HR0011831554 is acknowledged.

\section{AUTHOR CONTRIBUTIONS}

W.L., N.R., V.C. and A.F. conceived the project. W.L. deposited the multilayered films, with the help of S.C. and F.A.; W.L. and F.A. performed the magnetic characterization of the SAFs and optimization of the magnetic properties. W.L., D.M. and F.A. performed the MFM experiments, 
with the help of K.B. and A.V.; W.L. performed the micromagnetic simulations. W.L., N.R. and V.C. prepared the manuscript, and all authors discussed and contributed to the final manuscript.

\section{COMPETING INTERESTS}

The authors declare no competing interests. 


\section{FIGURES}

a

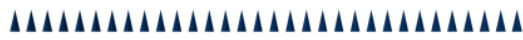
WWWWWWWWWWWWr

b

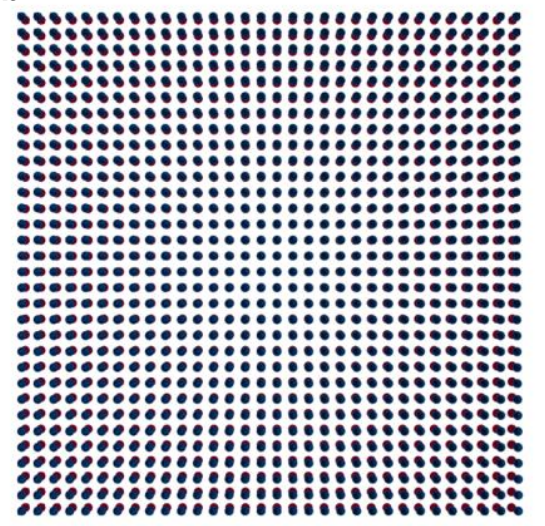

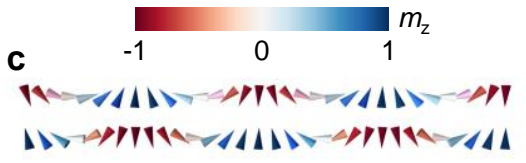

d

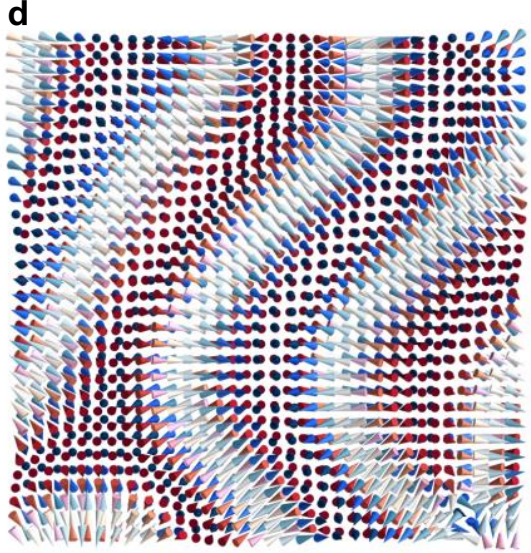

e

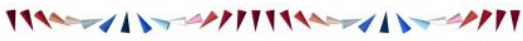

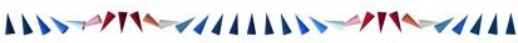

f

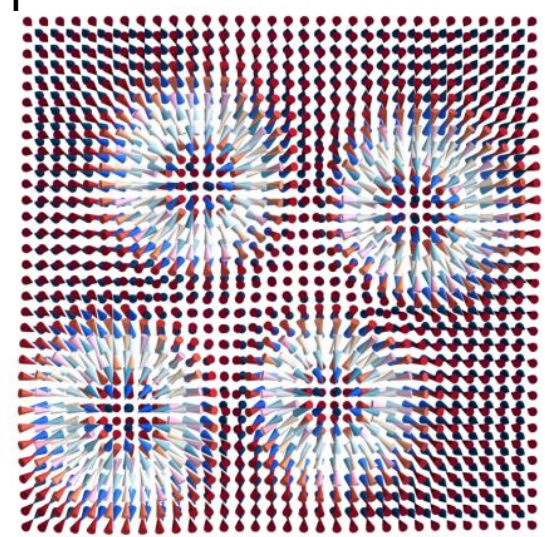

FIG. 1. Synthetic antiferromagnet $(\mathrm{SAF})$ in different configurations. a. Side view of the magnetic order in the SAF, for a large effective perpendicular magnetic anisotropy (PMA). b. Top view of the two antiferromagnetically coupled and saturated layers, for a large effective PMA. c. Side view of a magnetic spin-spiral in the SAF, for a vanishing effective PMA. d. Top view of the two antiferromagnetically coupled layers, hosting disordered spin-spirals, for a vanishing effective PMA. e. Side view of a biased magnetic spin-spiral in the SAF for a vanishing effective PMA. f. Top view of the two antiferromagnetically coupled layers, hosting antiferromagnetic skyrmions, for a SAF with vanishing effective PMA and subject to an up-pointing biasing interaction field in the bottom layer. The cones show local magnetization direction in the layers, whose color specifies the vertical component of the magnetization, $m_{\mathrm{z}}$, according to the colorscale. 

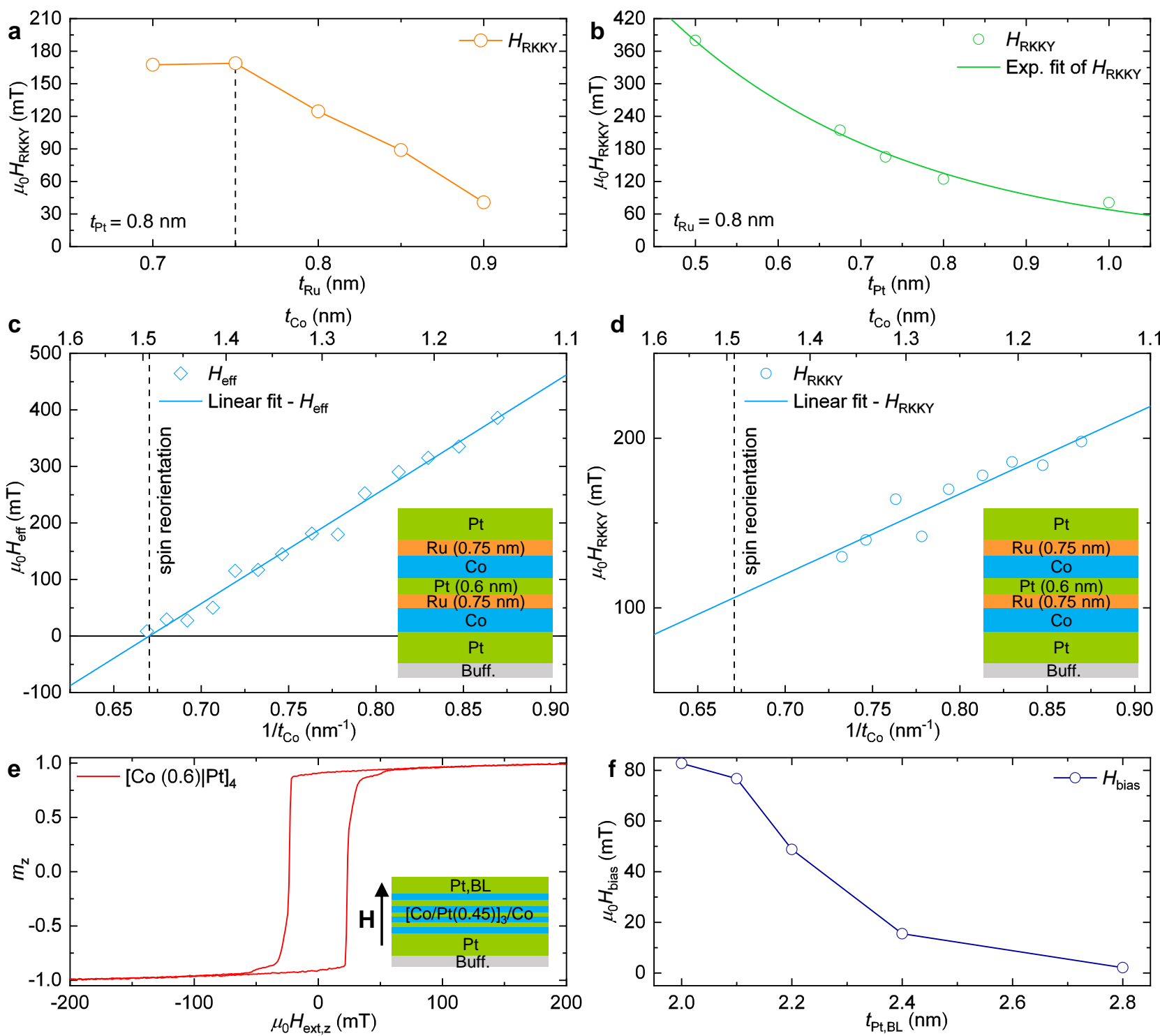

FIG. 2. Elaboration of $[\mathrm{Pt} / \mathrm{Co} / \mathrm{Ru}]$ multilayers and bias layer $(\mathrm{BL})$ to obtain an optimized biased synthetic antiferromagnet (SAF). a,b. Ruderman-Kittel-Kasuya-Yoshida (RKKY) coupling field $\mu_{0} H_{\mathrm{RKKY}}$ as a function of Ru layer thickness $t_{\mathrm{Ru}}$ for Pt layer thickness $t_{\mathrm{Pt}}=0.8 \mathrm{~nm}$ (a) and as a function of $t_{\mathrm{Pt}}$ for $t_{\mathrm{Ru}}=$ $0.8 \mathrm{~nm}$ (b). Dashed line in panel a locates the maximum of RKKY field. The solid line curve in panel b is a fit of the data points to an exponential decay function $\mu_{0} H_{\mathrm{RKKY}}\left(t_{\mathrm{Pt}}=0\right) \mathrm{e}^{-t_{\mathrm{Pt}} / t_{\mathrm{d}}}$ with typical attenuation length $t_{\mathrm{d}}=0.30 \mathrm{~nm}$. c,d. Effective perpendicular magnetic anisotropy (PMA) field $\mu_{0} H_{\text {eff }}$ (c) and RKKY coupling field $\mu_{0} H_{\mathrm{RKKY}}(\mathrm{d})$ as a function of inverse Co layer thickness $1 / t_{\mathrm{Co}}$. The lines are linear fits of the data points. The spin reorientation occurs at around $t_{\mathrm{Co}}=1.49 \mathrm{~nm}$ (dashed vertical line in panels $\mathrm{c}$ and d). e. Out-of-plane magnetization $m_{\mathrm{z}}$ loop for a $\mathrm{BL}$ with $t_{\mathrm{Co}}=0.6 \mathrm{~nm}$ and $t_{\mathrm{Pt}}=0.45 \mathrm{~nm}$, as a function of perpendicular external field. f. Coupling field $\mu_{0} H_{\mathrm{bias}}$ in the BL-SAF as a function of $t_{\mathrm{Pt}, \mathrm{BL}}$. The insets in $\mathrm{c}, \mathrm{d}$ and e depict the multilayer geometry and measurement configuration. 

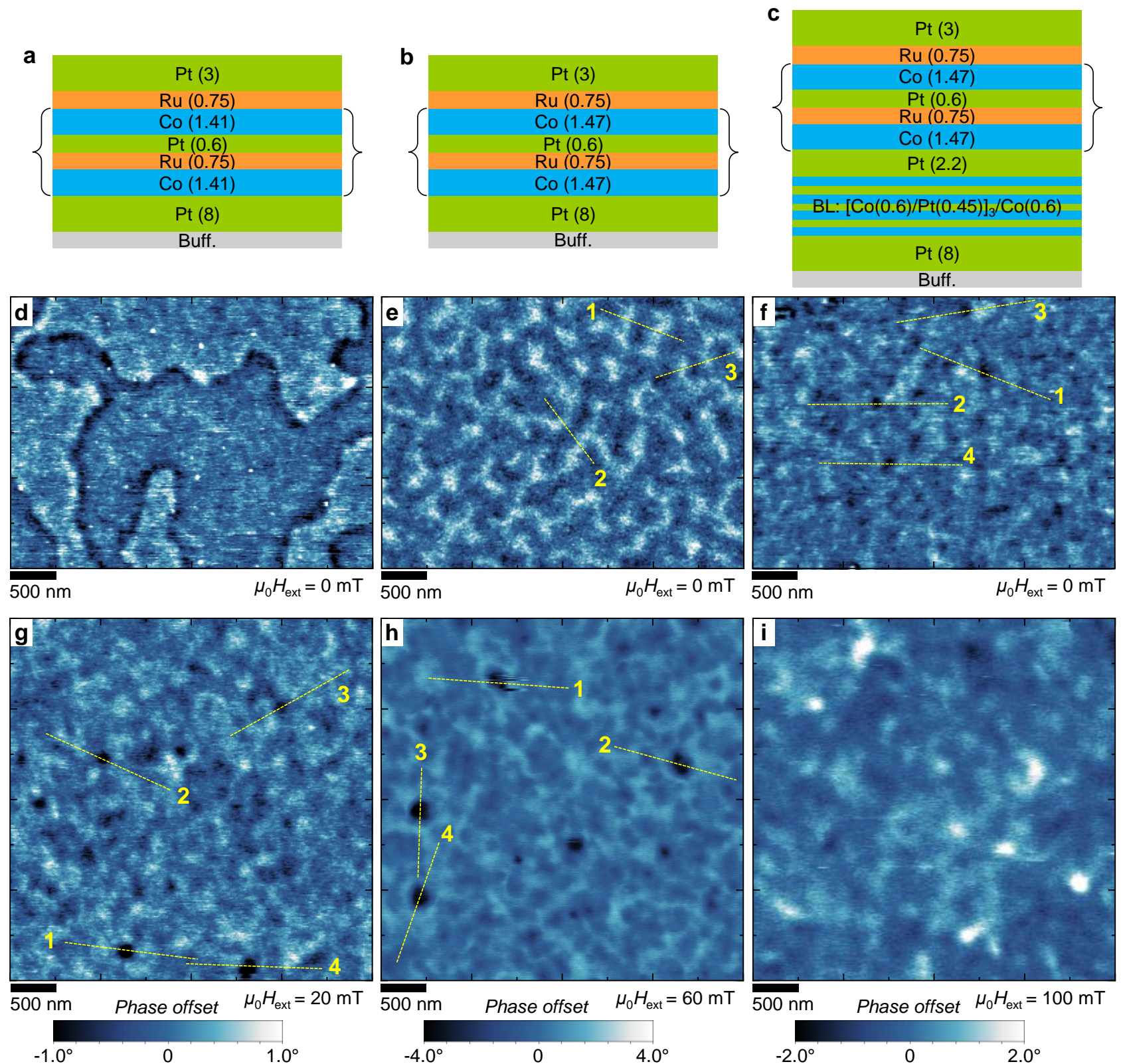
FIG. 3. Magnetic Force Microscopy (MFM) observation of magnetization textures in synthetic antiferromagnets (SAFs). a-c. Multilayer stack for SAF with significant effective perpendicular magnetic anisotropy (PMA) (a), SAF with vanishing effective PMA (b) and bias layer (BL)-SAF system (c). Brackets indicate the SAF system. Note that indeed, $t_{\mathrm{Co}}=1.415 \mathrm{~nm}$ is well below, while $t_{\mathrm{Co}}=1.47 \mathrm{~nm}$ is very close to the spin reorientation thickness previously determined. d. MFM image of SAF with effective PMA at $0 \mathrm{mT}$. e. MFM image of SAF with vanishing effective PMA at $0 \mathrm{mT}$. f. MFM image of BL-SAF at $0 \mathrm{mT}$, after saturation of the BL under an external perpendicular field $\mu_{0} H_{\text {ext }}=60 \mathrm{mT}$. $\mathbf{g}-\mathbf{i}$. MFM observations of the BL-SAF system under external applied perpendicular field for $\mu_{0} H_{\text {ext }}=20 \mathrm{mT}(\mathrm{g})$, for $\mu_{0} H_{\text {ext }}=60 \mathrm{mT}$ (h) and for $\mu_{0} H_{\text {ext }}=100 \mathrm{mT}$ (i). All MFM images in panels $\mathrm{d}-\mathrm{g}$ share the same color scale shown below panel g, while MFM images in panels h and i use different scales, all indicating the phase offset in lift mode. Yellow dashed lines in panels e- $\mathrm{h}$ indicate line sections of the MFM images, which are shown in Figs. $4 \mathrm{p}-\mathrm{d}$. 

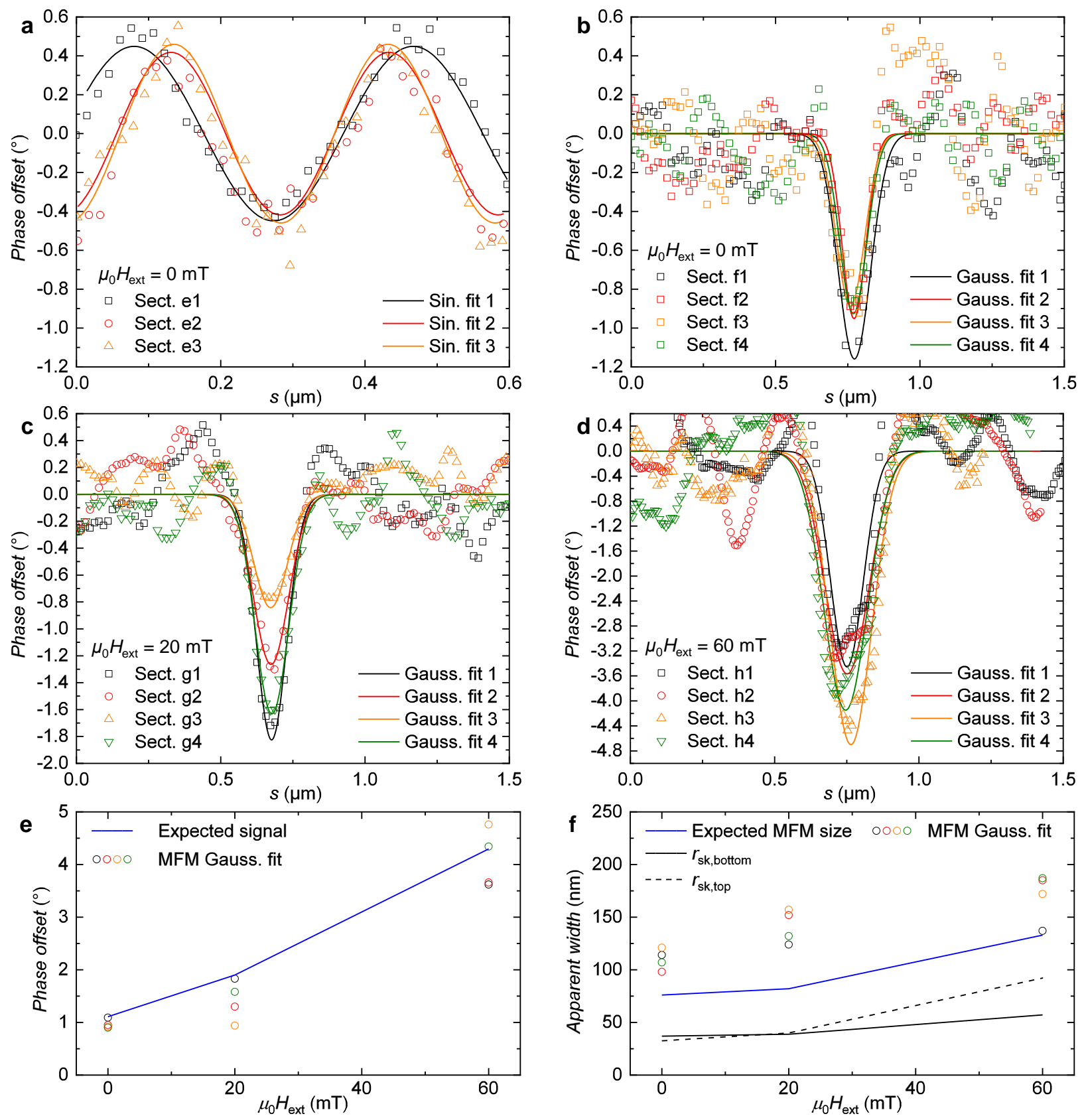
FIG. 4. Quantitative analysis of Magnetic Force Microscopy (MFM) signal (phase offset in lift mode). a. Line sections e1, e2 and e3 of the MFM signal in the image of the spin-spiral, Fig. 3 . Solid line curves are fits of the measurement points to simple sinusoids, to extract periodicity. b. Line sections f1, f2, f3 and $\mathrm{f} 4$ of the MFM signal in the image of the antiferromagnetic skyrmions at zero field, Fig. 35. c. Line sections g1, g2, g3 and $\mathrm{g} 4$ of the MFM signal in the image of the antiferromagnetic skyrmions at $\mu_{0} H_{\text {ext }}=$ $20 \mathrm{mT}$, Fig. 35. d. Line sections h1, h2, h3 and h4 of the MFM signal in the image of the antiferromagnetic skyrmions at $\mu_{0} H_{\text {ext }}=60 \mathrm{mT}$, Fig. 3 3 . In panels b, c and d, solid line curves are fits of the measurement points to Gaussian peak functions. The horizontal axis of these line sections panels is the distance along each corresponding line in Fig. 3 e,f. Comparison of contrast level (e) and apparent skyrmion diameter [defined as the full width at half maximum (FWHM)] of the peaks (f) between calculations (blue lines) and MFM data (colored dots). Black lines in panel f correspond to skyrmion diameters $2 r_{\text {sk }}$ in the bottom (solid line) and top (dashed line) layers, as determined by the simulation using experimental parameters. 

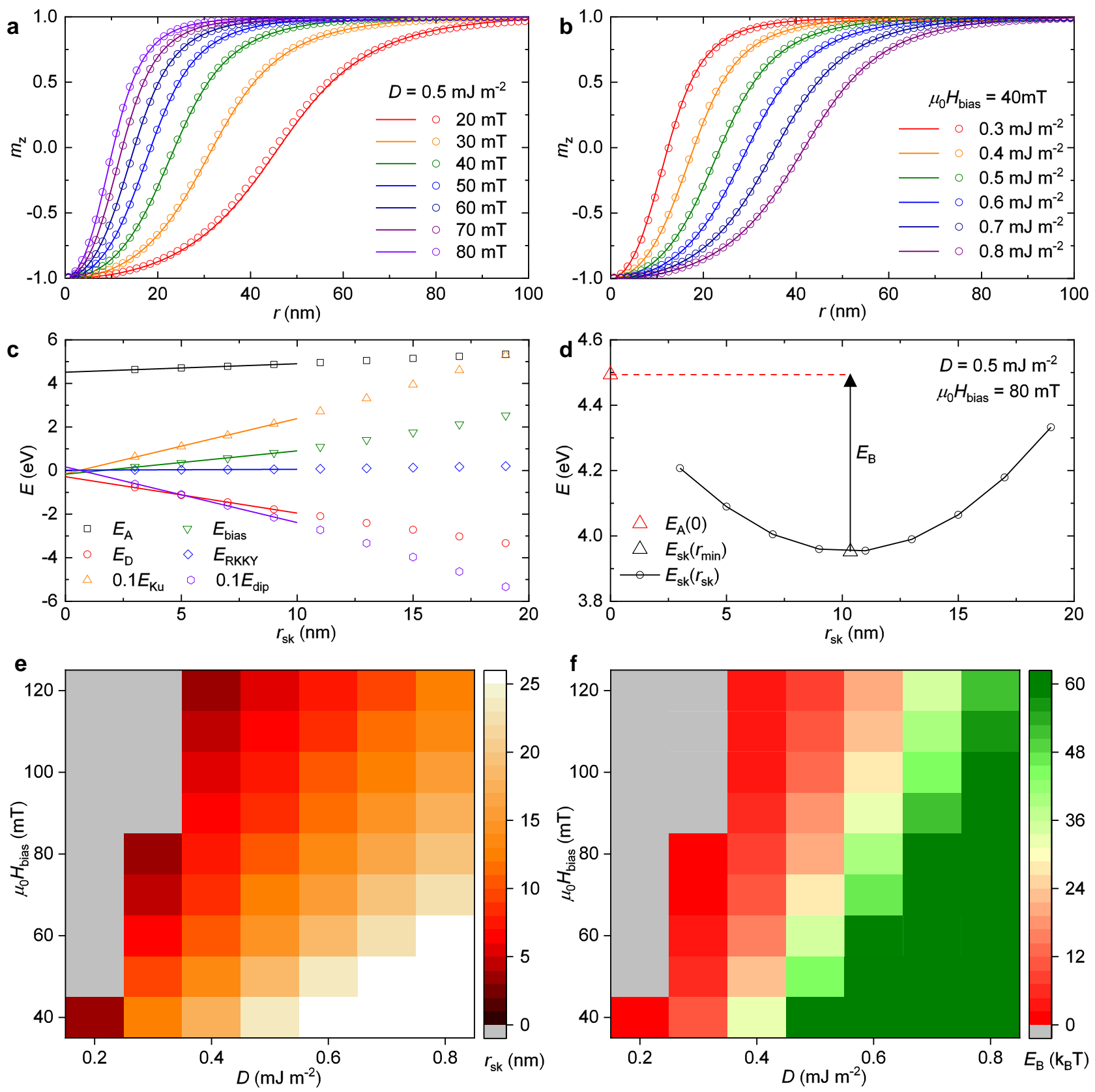
FIG. 5. Evaluation of sizes and energies of antiferromagnetic skyrmions in the bias layer (BL)-synthetic antiferromagnet (SAF) system. a,b. Radial profiles of the vertical magnetization component $m_{\mathrm{z}}$ of the skyrmion in the bottom layer for $\mu_{0} H_{\text {bias }}=20-80 \mathrm{mT}$ with $D=0.5 \mathrm{~mJ} \mathrm{~m}^{-2}$ (a), and for $D=0.3-0.8 \mathrm{~mJ} \mathrm{~m}^{-2}$ with $\mu_{0} H_{\text {bias }}=40 \mathrm{mT}$ (b). Colored lines are results from our model while circles are the results from $\mathrm{MuMax}^{3}$ software 42. c. Evolution of the 6 energy terms, namely, exchange energy $E_{\mathrm{A}}$, Dzyaloshinskii-Moriya interaction (DMI) energy $E_{\mathrm{D}}$, perpendicular magnetic anisotropy (PMA) energy $E_{\mathrm{Ku}}$, BL coupling energy $E_{\text {bias }}$, Ruderman-Kittel-Kasuya-Yoshida (RKKY) energy $E_{\mathrm{RKKY}}$, and dipolar energy $E_{\text {dip }}$, contributing to the total skyrmion energy with skyrmion size. Note that all energy terms are defined in the way that they reach zero in the uniform, antiferromagnetic state. The dipolar energy term essentially compensates the PMA energy, due to $K_{\text {eff }}=0$. Lines are linear fits of the different energy terms at low radius values. d. Evolution of the total skyrmion energy $E_{\mathrm{sk}}$ with skyrmion size. Red and black triangles indicate the energy at zero radius and minimal energy radius, respectively. Their difference is the energy barrier $E_{\mathrm{B}}$, as denoted by the arrow. In panels $\mathrm{c}$ and $\mathrm{d}, D=0.5 \mathrm{~mJ} \mathrm{~m}^{-2}$ and $\mu_{0} H_{\text {bias }}=80 \mathrm{mT}$. e,f. Skyrmion radius $r_{\mathrm{sk}}$ in the bottom layer (e) and energy barrier $E_{\mathrm{B}}$ (f) as a function of $D$ and $\mu_{0} H_{\mathrm{bias}}$, given by the color scales on the right. A thermal stability barrier higher than $40 k_{\mathrm{B}} T$ is typically required for long-term storage of skyrmions, corresponding to the green color in the diagram. Gray color indicates parameters for which the antiferromagnetic skyrmions spontaneously collapse, that is, for which the energy barrier allowing metastability is absent. 